\title{
ESTUDIO ETNOBOTÁNICO DE PLANTAS USADAS EN LA ALIMENTACIÓN DE LOS CAMPESINOS DEL NOROESTE DE LA PAMPA ARGENTINA
}

\author{
ETHNOBOTANICAL STUDY OF FOOD PLANTS USED BY FARMERS \\ FROM THE NORTHWESTERN ARGENTINIAN \\ LA PAMPA REGION
}

Walter Alejandro Muiño ${ }^{1}$

\begin{abstract}
En este trabajo se registran las especies vegetales involucradas en la alimentación de un grupo humano actual, en este caso la población del noroeste de La Pampa (Argentina) conformada por campesinos criollos descendientes de colonos inmigrantes. El tema se abordó desde la perspectiva etnobiológica, destacando la presencia y el papel de las plantas accesibles en la zona. En tal sentido, se resaltan aspectos de interés propios de la etnobotánica como son la nomenclatura vernácula de las especies, las comidas y bebidas típicas, las formas de apropiación, dando cuenta en todos los casos del estado de persistencia de su empleo y los cambios sufridos hasta la actualidad. Se registró un total de 62 taxones vegetales que intervienen en la alimentación de esta población, información que constituye un novedoso aporte al conocimiento de la etnobotánica regional y de zonas áridas americanas.
\end{abstract}

Palabras claves: conocimiento tradicional, alimentación, etnobotánica, recolección, agricultura.

This study documents the plants consumed by the modern inhabitants of the Northwestern area of La Pampa (Argentina), creole farmers descended from colonial immigrant. An ethnobiological approach is applied which highlights the presence and role of locally available plants. As such, the vernacular names of species, traditional meals and drinks, and forms of exploitation were recorded, examining the persistence of use and its changes through time. A total of 62 plant taxa associated with the diet of this population was recorded, contributing new knowledge of local ethnobotany and that of arid zones in the Americas.

Key words: Traditional knowledge, diet, ethnobotany, collection practices, agriculture.

En Sudamérica, los estudios etnobotánicos vinculados a la alimentación presentan un interés particular ya que esta región posee un alto porcentaje de endemismos que se encuentran amenazados por los procesos de degradación del ambiente (Scarpa 2009).

El presente trabajo forma parte de una investigación etnobotánica que hace referencia a los conocimientos tradicionales de una comunidad campesina actual que habita el extremo oeste de la provincia de La Pampa (Muiño 2010a). Esta región corresponde al área con menor precipitación anual de la región pampeana, que junto con la intensa evapotranspiración confiere a este ambiente condiciones de aridez muy marcadas. Las condiciones climáticas descritas constituyen generalmente un fuerte límite para las prácticas agrícolas, permitiendo, en cambio, mejores oportunidades para las actividades pastoriles y de caza-recolección. Estas características han motivado que gran parte de las investigaciones en la región fueran hasta el presente llevadas a cabo sobre el área oriental más húmeda y productiva. Por tal razón existe un marcado desconocimiento sobre estos ecosistemas y los grupos humanos que viven en él.

En La Pampa, uno de los trabajos etnobotánicos más detallados sobre el papel de las plantas alimenticias nativas está referido a la comunidad ranquel, grupo aborigen que dominó el sector centro-norte de esta provincia argentina (Steibel 1997). Bajo este enfoque y con diferentes grupos humanos también se destacan otras investigaciones en regiones aledañas. Así, Martínez Crovetto (1968, 1982) documentó las plantas en la alimentación de los pueblos araucanos y pampas de la región bonaerense y patagónica. Ladio (2002, 2004), por

$1 \quad$ Facultad de Ciencias Exactas y Naturales, Universidad Nacional de La Pampa, Santa Rosa, La Pampa, Argentina. wmuino@yahoo.com.ar 
otra parte realizó contribuciones al tema estudiando poblaciones criollas y mapuches de Neuquén, y asimismo lo hicieron Arias Toledo et al. (2007) con criollos de Córdoba, Argentina. Por otra parte, la información relacionada con la cultura alimentaria en Argentina también ha sido documentada a través de estudios florísticos, etnográficos, históricos y antropológicos (Agüero Blanch 1967; Martinelli 2008; Rapoport et al. 2001; Remedi 1997; Ruiz Leal 1972). Si bien existen elementos en común con otras comunidades, los criollos que habitan el oeste pampeano presentan fisonomías propias que definen las estrategias de alimentación mejor adaptadas a sus características culturales y que no han sido estudiadas hasta el presente. Los resultados de este trabajo se presentan aquí como una contribución al conocimiento sobre los recursos vegetales vinculados a la alimentación de las sociedades rurales actuales de zonas áridas de la Argentina.

La población del área está constituida por campesinos descendientes de colonos, en su mayoría de origen español, que arribaron a la zona principalmente desde las provincias limítrofes de Mendoza y San Luis. Luego de 1879, ya finalizada la etapa conocida como "conquista del desierto" (Martínez Sarasola 1992), los nuevos colonos debieron implementar estrategias de producción que ya dominaban en sus lugares de origen así como otras nuevas adaptadas al ambiente. En este sentido, las condiciones climáticas, la disponibilidad de agua y las características del terreno actuaron muy frecuentemente como un freno para las experiencias agrícolas. La actividad que constituyó la base de la economía del lugar quedó por lo tanto condicionada a la actividad pastoril sedentaria en campos abiertos (Covas 1998) cuyo objetivo era la cría de ganado ovino, caprino, equino y en menor escala el bovino. Junto con estas características, las grandes distancias hacia los centros urbanos más importantes de la región y la red vial poco desarrollada dieron lugar a una situación de aislamiento relativo del área que influyó en las pautas de consumo alimentario, caracterizadas por una elevada ingesta de carnes en desmedro de los alimentos de origen vegetal.

Estos condicionamientos y las pautas culturales con fuerte arraigo en la actividad pastoril permiten suponer que aún subsiste una cultura alimentaria poco influida por los patrones externos a esta comunidad. En ella, los vegetales tienen una participación menor con respecto a los alimentos de origen animal, pero son un complemento importante en la dieta y forman parte de platos dulces y bebidas tradicionales. En función de ello, el objetivo de este estudio es realizar un registro de los recursos vegetales que intervienen en la dieta de esta población, sus métodos de obtención y las formas de conservación de los alimentos de origen vegetal, así como documentar la persistencia o abandono de estos elementos en la cultura local. La metodología implementada en este trabajo se encuadra dentro del enfoque cualitativo considerado como la estrategia que permite comprender mejor las motivaciones de la gente en su relación con las plantas.

\section{Materiales y Métodos}

El área de estudio está ubicada en el sector occidental del departamento Chicalcó en el NO de la provincia de La Pampa (Argentina), entre $36^{\circ} 00^{\prime}$ y $36^{\circ} 47^{\prime}$ de latitud S y $67^{\circ} 40^{\prime}$ y $68^{\circ} 20^{\prime}$ de longitud W (Figura 1). Esta área corresponde al ejido municipal de La Humada que cuenta con una población de 954 habitantes considerando la población urbana y rural (Instituto Nacional de Estadísticas y Censos 2001) y se constituye en un espacio político y administrativo de la vida local. Desde el punto de vista fitogeográfico corresponde a la provincia del Monte (Cabrera 1971) y en el sector austral incluye algunas porciones de la provincia de la Payunia (Martínez Carretero 2004).

Los trabajos de campo se realizaron entre agosto de 2005 y febrero de 2009. Para lograr un primer acercamiento al tema se llevaron a cabo entrevistas abiertas con pobladores que fueran residentes permanentes en el área de estudio. Se realizaron registros sobre las historias de vida, para detectar los informantes mejor calificados en el tema, los rasgos socioculturales emergentes, así como los procesos de abandono y persistencia de hábitos relacionados con el empleo de plantas en la alimentación. La información que alude a costumbres del pasado hace referencia a lo que acontecía hasta la década de 1970, época en la que se verificaron cambios que influenciaron en forma destacada la economía local (Comerci 2008). El total de los entrevistados ascendió a 69, compuesto por 27 mujeres y 42 varones, todos ellos adultos, cuya edad promedio fue de 56 años. El número de personas entrevistadas fue definido de acuerdo a la "Ley de rendimientos decrecientes" (Martin 2000). Todos los registros fueron realizados en forma 


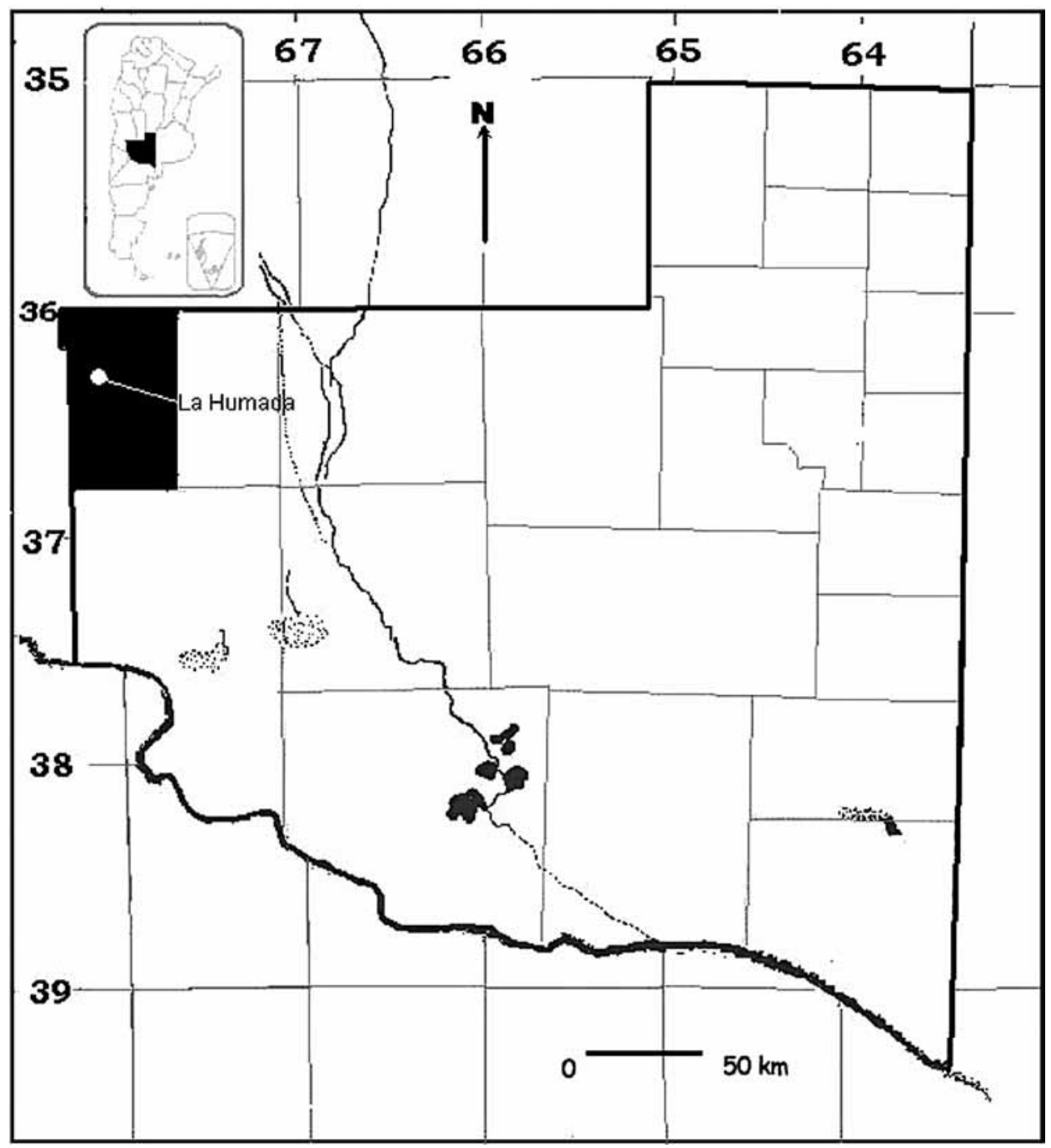

Figura 1. Mapa de la provincia de La Pampa donde se destaca el área de estudio y en ella la localidad cabecera de La Humada.

Map of the province of La Pampa, showing the study area and its capital, La Humada.

escrita. Junto con las entrevistas se realizaron 47 encuestas semiestructuradas en forma aleatoria y proporcional a las poblaciones rural y urbana con el fin de registrar la persistencia de los hábitos de recolección y cultivo de plantas.

Teniendo en cuenta que el área de estudio es una región con vegetación caducifolia, las colectas se concentraron en los viajes de primavera y verano. En los viajes realizados durante el otoño e invierno el trabajo se centró preferentemente en el registro de la información a partir de las entrevistas.
Las especies vegetales silvestres y cultivadas empleadas en la alimentación fueron documentadas a través de colectas de ejemplares, tarea en la que se contó con la colaboración de los informantes locales. El material fue herborizado, identificado taxonómicamente e incorporado a la colección del Herbario SRFA perteneciente a la Facultad de Agronomía de la Universidad Nacional de La Pampa. Los nombres científicos de las plantas silvestres fueron asignados de acuerdo al Catálogo de las Plantas Vasculares del Cono Sur (Zuloaga et al. 2008). Para los taxones adventicios se siguió 
la nomenclatura del United States Department of Agriculture (2011).

\section{Resultados}

\section{Recolección de plantas silvestres}

La recolección de recursos vegetales para la alimentación es una costumbre infrecuente entre los pobladores del oeste pampeano. Esta tarea está supeditada a ocasionales salidas de recolección en el campo o como actividad complementaria a las otras labores rurales del cuidado de los rodeos. Dentro del total de los taxones vegetales registrados en este estudio (62), el 23\% corresponde a especies recolectadas (Tabla 1). La actividad más importante en ese rubro está representada por la colecta de los frutos de piquillín (Condalia microphylla). Las pequeñas drupas se suelen consumir como fruta fresca pero también es frecuente elaborar arrope con ellas.

Las técnicas de extracción son la recolección manual directa o la que consiste en colocar un cuero o lona debajo del árbol y golpear sus ramas con un palo para provocar la caída de sus frutos. Si bien la técnica de extracción manual directa es más lenta y dificultosa, muchos pobladores la prefieren porque sostienen que provoca menos daño a la planta, de modo tal que pueden obtener buenas producciones todos los años. Ellos aseguran que con el otro método la planta disminuye drásticamente su producción de fruta en los años siguientes.

Los tubérculos del chaquil o macachín (Arjona tuberosa) también son recolectados para el consumo, pero los informantes de edad más avanzada indicaron que esta actividad era más frecuente en el pasado y actualmente se está abandonando. Los testimonios indican que estas "papillas" son más abundantes en lugares arenosos, principalmente entre las matas de tomillo macho (Junellia seriphioides) y de yerba de oveja (Baccharis darwinii). Los tubérculos, de sabor dulce, se pueden consumir directamente pero también pueden molerse y agregar al agua del mate para endulzarla o bien prepararlos en forma de arrope, aunque en la actualidad no se registró la elaboración de esta receta y solo se recolecta en forma ocasional.

Los árboles y arbustos del género Prosopis proveen frutos cuya recolección aún persiste. Tanto el algarrobo (Prosopis flexuosa f. flexuosa) como el alpataco (Prosopis flexuosa var. depressa) proveen legumbres que la gente consume directamente más como tentempié o refrigerio que como alimento principal. Su consumo en preparaciones como el patay, la aloja, o el arrope es una práctica que se está perdiendo en el área, pero que los ancianos recuerdan como muy común en su infancia. Para realizar estas preparaciones eran más buscados los frutos del algarrobo que los de alpataco debido a que aquellos son más dulces.

Los frutos del albaricoque (Ximenia americana), chañar (Geoffraea decorticans), penca (Opuntia sulphurea) y solupe frutero (Ephedra ochreata) son consumidos en forma directa, pero hubo menos consenso en su actividad de recolección en comparación con las anteriores especies. Con ellos, a excepción del primero, también se elaboraba arrope en épocas pasadas.

Además de las mencionadas existen otras especies que si bien no constituyen alimentos en el sentido estricto, intervienen en el uso culinario como condimentos y saborizantes. Tal es el caso del tomillo (Acantholippia seriphioides), del que algunos utilizan la parte aérea para condimentar la carne asada de piches (Zaedyus pichiy) y otros animales silvestres. El mismo uso le dan a la yerba del lagarto (Acantholippia seriphioides), una variedad de tomillo que presenta una fragancia más delicada que la anterior con la cual se saboriza el mate. También son empleadas con este fin el chilchil (Tagetes mendocina) y el té pampa (Thelesperma megapotamica), pero es costumbre agregar a esta bebida una amplia diversidad de plantas (Tabla 1), que además de modificarle el sabor, actúan como digestivos y contribuyen con la buena salud.

Los resultados expresan que la totalidad de la población entrevistada conoce plantas comestibles del monte y aunque varían las especies mencionadas, el piquillín (Condalia microphylla) estuvo presente en todas las respuestas. Entre la población que no practica la recolección, las razones que justifican tal comportamiento expresan la falta de motivación, el desabrimiento de las frutas del monte, y la escasez de frutos en algunos lugares. La preferencia por la adquisición de frutas y hortalizas, así como de mermeladas y otros alimentos manufacturados en los comercios de La Humada también fue un indicador de la falta de motivación hacia esta práctica. En general, cualquier integrante del grupo familiar se encarga de las tareas de recolección, sin embargo es muy frecuente que esta función la lleven a cabo los niños, en tanto 
que la elaboración de productos como el arrope o el patay la realizan mayoritariamente las mujeres adultas del hogar.

\section{Cultivo en huertas familiares}

Las experiencias de cultivos domésticos de hortalizas y árboles frutales son limitadas. Hasta hace pocas décadas, el abastecimiento de hortalizas se realizaba principalmente a partir de las pequeñas huertas hogareñas y de los vendedores ambulantes que transitaban el área rural. En la región, la horticultura no ha logrado el impulso que tuvo en la provincia limítrofe de Mendoza con sus amplias áreas de regadío. La ausencia de cursos de agua superficiales y la limitada oferta subterránea condicionan esta actividad. Aún hoy, en las huertas hogareñas de La Humada no existen adecuados sistemas de irrigación.

Del total de individuos encuestados, solo el $13 \%$ practica el cultivo de huertas. Los pobladores justifican esta carencia en la escasez de agua para el cultivo, pero también manifestaron otras razones como falta de motivación, mala calidad de la tierra para el cultivo, falta de predios aislados de los animales y poca disponibilidad de tiempo para la actividad. Actualmente las mejores condiciones para el comercio de alimentos permiten el acceso a frutas y verduras frescas en La Humada, además de las compras que se realizan directamente a través de vendedores ambulantes en los predios rurales. Sin embargo, los centros urbanos de la provincia de Mendoza proveen plantas que permiten experiencias de cultivo con buenos resultados en huertas y patios. Entre los frutales las especies más cultivadas son perales (Pyrus communis), durazneros (Prunus persica), damascos (Prunus armeniaca), vides (Vitis vinifera) y membrilleros (Cydonia oblonga). Los cultivos más frecuentes en las huertas son zapallos (Cucurbita pepo), maíz (Zea mays), lechuga (Lactuca sativa), remolacha (Beta vulgaris subsp. macrocarpa), acelga (Beta vulgaris subsp. vulgaris), sandía (Citrullus lanatus subsp. vulgaris), rabanito (Raphanus sativum), zanahoria (Daucus carota), y tomate (Solanum lycopersicum).

En las huertas también se emplea el espacio para cultivar especias aromáticas. Las especies más cultivadas son el orégano (Origanum vulgare), menta (Mentha sp.), ajo (Allium sativum), perejil (Petroselinum crispum), albahaca (Ocimum basilicum), ajenjo (Artemisia absinthium), y té de burro (Aloysia polistachya). En la Tabla 1 se presentan 29 taxones vegetales cultivados con fines alimentarios, que constituyen el $47 \%$ del total de las especies aquí registrado.

\section{Los vegetales en las comidas}

Si bien los platos suelen ser variados, las comidas más frecuentes son las carnes asadas. Estas proceden del ganado doméstico y de los animales silvestres obtenidos en las cacerías. Generalmente esta preparación se realiza en fogones del interior de la vivienda, que además se emplean para la calefacción del hogar, o en hornos de barro instalados en el exterior.

Además de la forma asada, la carne forma parte de muchos otros platos ampliamente difundidos en la cocina tradicional como los locros, pucheros, guisos y carbonadas. En estos también intervienen ingredientes de origen vegetal como el maíz (Zea mays), arroz (Oryza sativa), tomate (Solanum lycopersicum), zapallos (Cucurbita pepo, C. maxima), camote (Ipomoea batatas), papa (Solanum tuberosum), zanahoria (D. carota) y cebolla (Allium cepa). Se registró una variante de estofado local denominado printincao, que se prepara con papa y abundante choclo, este puede incluir porotos (Phaseolus vulgaris), zapallo, cebolla, pero nunca carne.

Los condimentos más utilizados son el ajo ( $A$. sativum) y el ají (Capsicum annum), pero también se emplean con frecuencia otros como el pimentón (C. annum), el laurel (Laurus nobilis) y la pimienta (Piper nigrum) que se compran en el comercio a excepción del ajo, que en ocasiones se cultiva en las huertas.

Entre las comidas tradicionales que han perdido vigencia se puede mencionar al mote, un plato que se elabora con granos de trigo o de maíz. En su cocción pueden intervenir cenizas de pichana (Baccharis spartioides) que actúan como acelerador de la cocción. El ñaco se prepara localmente tostando granos de maíz (Zea mays) o trigo (Triticum aestivum) sobre arena colocada en el fondo de una olla hasta que estos estallan. Con el maíz partido también se cocina el plato tradicional denominado mazamorra.

El pan se cocina en un hornillo hecho en un pozo de algunos centímetros en el suelo. En su elaboración se emplea grasa y el leudante utilizado en la 


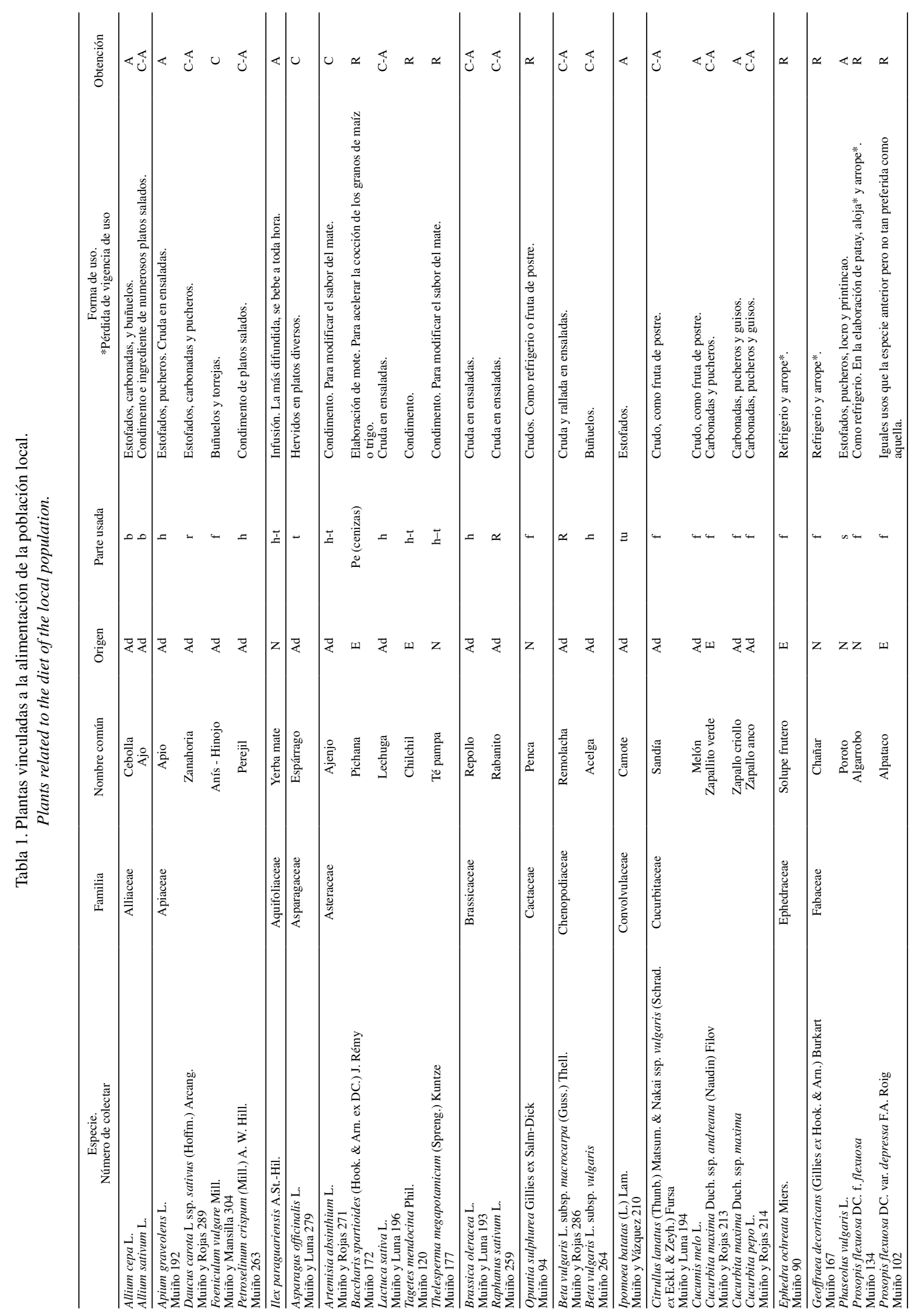




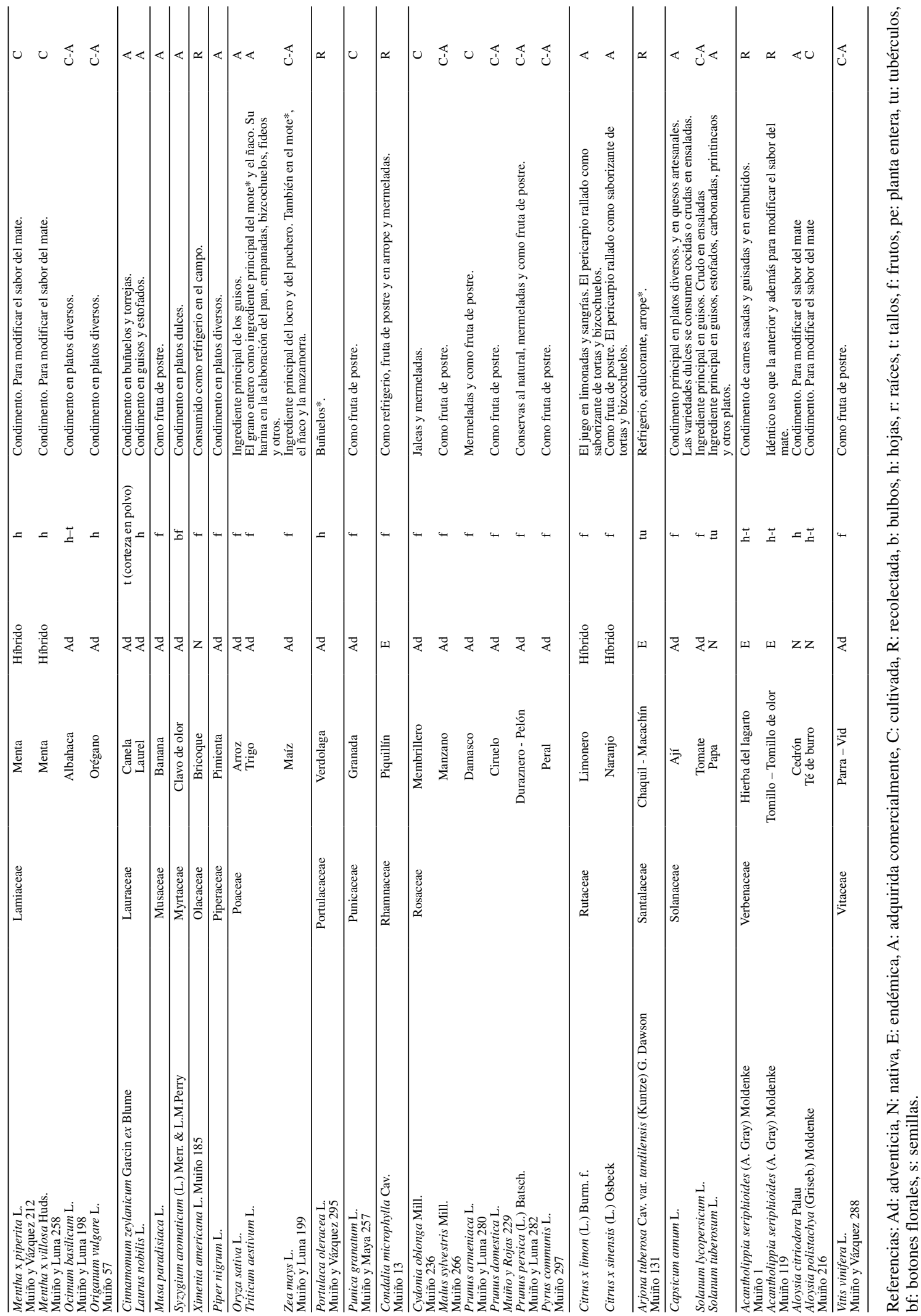


masa estaba constituido en el pasado por una mezcla fermentada de harina con agua. Actualmente este se reemplaza por levadura comercial. Como variante, la "fariña" es una clase de harina de trigo más gruesa que en ocasiones se mezclaba con cebolla picada (Allium cepa) y grasa de piche (Zaedyus pichiy) y luego se consumía asada como un sustituto del pan. Actualmente esta comida se encuentra en desuso.

Otro plato tradicional que en la actualidad está perdiendo vigencia es el patay. En el área se prepara moliendo y tamizando legumbres de algarrobo (Prosopis flexuosa f. flexuosa) o alpataco (Prosopis flexuosa var. depressa). Se señaló que con la fracción gruesa que queda remanente luego del tamizado se pueden hacer aloja y arrope.

A diferencia del anterior está muy difundida la elaboración de buñuelos, torrejas y tortas fritas, para acompañar desayunos y meriendas. Estos platos se suelen condimentar con especias tales como clavo de olor (Syzygium aromaticum), canela (Cinnamomum zeylanicum), esencia de vainilla y anís (Foeniculum vulgare), pero también existe la variante salada que lleva pimiento (Capsicum annum) y cebolla (A. cepa). Estos platos se elaboran con harina de trigo, pero en los buñuelos también se suelen emplear verduras cocidas que se indican en la Tabla 1.

\section{Las bebidas}

La bebida popular con mayor arraigo es el mate, al que es costumbre agregar otras plantas, que además de modificar su sabor, actúan saludablemente sobre el organismo. Los niños también beben mate, pero con más frecuencia ingieren, además de leche, una infusión de yerba mate (Ilex paraguariensis) más diluida que localmente se conoce como "yerbeado".

El vino era y sigue siendo una bebida de consumo importante, en especial durante fiestas y reuniones, en las cuales se preparaban sangrías y cócteles a partir del vino. En la primera se diluye el vino con agua y se añade azúcar a gusto. A diferencia de ella, el "cóctel" se elabora mezclando dos yemas de huevo y azúcar en un litro de vino. Cuando la mezcla se hace homogénea se le añade más vino y azúcar hasta el gusto deseado.

La aloja es una bebida que no reportó registros de elaboración actual. Se indicó que era preparada a partir de la fracción gruesa remanente del cernido que se efectuaba luego de moler los frutos de algarrobo (Prosopis flexuosa f. flexuosa) y de alpataco
(P. flexuosa var. depressa). Esta fracción se diluía en agua, se dejaba decantar y se bebía directamente sin que medie proceso alguno de fermentación.

Entre los licores de elaboración casera se registró el preparado en base a una infusión de té pampa (Thelesperma megapotamica) que se mezcla con alcohol y azúcar quemada y junto con éste la "chupilca", que se elabora mezclando coñac con huevo. La "curillena", por ora parte, era una bebida espesa que se lograba mezclando ñaco con vino y azúcar, y que adopta este nombre porque se trata de una bebida que emborracha y que sacia el apetito. No obstante, también hubo registros de esta preparación con el nombre de chupilca y su elaboración es similar a la chupilca chilena. Las bebidas alcohólicas indicadas anteriormente han perdido vigencia de uso y en la actualidad son reemplazadas por las que se adquieren en los comercios.

\section{La conservación de los alimentos}

Entre las técnicas de conservación de alimentos de origen vegetal se pueden mencionar los arropes y mermeladas. Ambos métodos se basan en el principio de evaporar el agua, solo concentrando los azúcares de los vegetales para el caso de los arropes, y añadiendo azúcar en las mermeladas. Sin embargo, esta diferencia conceptual aún vigente en otras partes del país no tiene su correspondencia en el oeste pampeano, donde, salvo pocas excepciones, se suele añadir azúcar en la preparación de ambas conservas. Aunque se registró la elaboración de arropes a partir de diversos recursos vegetales silvestres en el pasado, actualmente casi todos ellos se encuentran en vías de desaparición, persistiendo solo el de piquillín (Condalia microphylla). Entre los recursos que han perdido vigencia de uso para este fin se destacan los frutos de solupe frutero (Ephedra ochreata), chañar (Geoffroea decorticans) y los tubérculos de chaquil (Arjona tuberosa).

\section{Discusión}

La base de datos del herbario SRFA y la información de los censos de vegetación registrados en la zona (INTA et al. 2004; Troiani et al. 1994) estiman para el área de estudio un total de 294 taxones específicos. De acuerdo a los resultados aquí expuestos, solo un $4,7 \%$ de las especies vegetales silvestres se estarían utilizando con fines alimentarios. Aun si se compara con los 145 taxones registrados en el área 
para todos los demás usos además de los alimentarios (Muiño 2010a), este porcentaje alcanzaría casi el $10 \%$. Por otra parte, en base a la información del Catálogo de las Plantas Vasculares del Cono Sur (Zuloaga et al. 2008), el $31 \%$ del total de las plantas que se presentan en este trabajo corresponden a taxones nativos, dentro de los cuales cerca de la mitad está constituida por endemismos.

La recolección de alimentos vegetales fue de importancia fundamental para los pueblos nativos orientada al sustento cotidiano y a la obtención de reservas alimentarias para tiempos de escasez (Borrero 2002; Prieto 1997-98). No obstante, los colonos la adoptaron como complemento de otros recursos que ya formaban parte de sus hábitos alimentarios, cuya base fundamental eran la carne de los animales domésticos y las hortalizas cultivadas, característica que es común en las comunidades agroganaderas modernas (Hilgert 1999).

Como ocurre en otras áreas circundantes (Böhm de Saurina 1961), la escasa actividad hortícola está relacionada con la poca disponibilidad de agua, hecho que condiciona las prácticas de cultivo a experiencias esporádicas en los años lluviosos, época en la que se producen recargas adecuadas de los acuíferos. Junto con ello la pérdida paulatina de la actividad hortícola se acentúa por el precio accesible y fácil disponibilidad de hortalizas en el comercio.

La descripción de las distintas variantes culinarias, los ingredientes que intervienen y las estrategias de conservación de alimentos del área están altamente influidas por el patrimonio cultural de la cocina criolla con fuerte presencia a su vez de la cocina española al igual que sucede en otras provincias mediterráneas de Argentina (Remedi 1997). Aquí está bien representada a través de platos como pucheros y carbonadas e ingredientes como la cebolla, el ajo y el laurel. También se observan aquí las modificaciones de la cocina criolla sobre la variante española a través del empleo de insumos tales como porotos y maíz en reemplazo de los garbanzos en el puchero (Remedi 1997). En cuanto a las especias, la mayoría son adquiridas comercialmente, y solo unas pocas son cultivadas en las huertas. $\mathrm{Al}$ respecto se pone de manifiesto el uso de aquellas que se emplean en toda la región pampeana, tanto en platos dulces como salados. Esto denota una diversificación menor comparada con otras comunidades como por ejemplo la puneña, en la que se utiliza un conjunto de condimentos muy variados (Scarpa y Arenas 1996). Esto expresaría una diferencia cultural marcada, ya que en el oeste pampeano no se observan estas ni otras características típicas de la cocina del altiplano destacadas por estos autores, como son los sabores, aromas y colores intensos, sino que aquí su carácter distintivo es la simplicidad.

Otra vinculación con la cultura criolla está representada por la presencia del arrope preparado con frutos de plantas silvestres en el arte culinario del oeste pampeano. El arrope, alimento de origen árabe, fue introducido en América por los españoles y su consumo coincide con el área de difusión de los cultivos de vides, de cuyos frutos se elaboraban los arropes más apreciados en tiempos de la conquista y colonización española (Scarpa 1999). Siguiendo las observaciones de este autor, es llamativa la gran variedad de frutos empleados en la elaboración de arrope en el NOA y probablemente debido a ello, el de piquillín sea menos apreciado que otros (Jolís 1972 [1789]). Sin embargo, en La Pampa el arrope de piquillín es actualmente el único que continúa vigente ya que constituye una de las pocas plantas en el área con disponibilidad de frutos suficiente y apta para la preparación de este alimento. Las técnicas de recolección aquí registradas son similares a las que se observan en otras áreas del país (Biurrun et al. 2007; Scarpa 1999).

Aunque los entrevistados aseguraron que el auténtico arrope se elabora sin edulcorar como se indica en las recetas tradicionales del norte de Argentina (Scarpa 1999), en la mayoría de los hogares visitados se confirmó el agregado de azúcar en su receta. Si la elaboración del arrope está vinculada con el grado de persistencia de la economía tradicional (Scarpa 1999) y su discontinuidad de empleo como técnica de conservación se relaciona con procesos de erosión cultural, se puede entonces deducir que la alteración de la receta tradicional y la desaparición del arrope elaborado con otras plantas silvestres de la región pampeana como el solupe frutero (Ephedra ochreata), chañar (Geoffroea decorticans) o chaquil (Arjona tuberosa) es una señal de este proceso.

La vinculación con la cultura alimentaria aborigen queda demostrada a través de los productos elaborados a partir de los frutos de árboles del género Prosopis (Capparelli 2007). No obstante, se evidencian procesos erosivos de este conocimiento ya que en la actualidad se prepara solo en forma ocasional y los testimonios de la elaboración y consumo de patay y de aloja fueron narrados por los ancianos como recuerdos de su infancia. En 
relación con esta última se observan diferencias en la forma de elaboración con otras regiones del país, en las cuales se trataría de una bebida alcohólica, recibiendo el nombre de añapa la misma bebida no fermentada (Capparelli 2007). Sin embargo, también existen evidencias de que bajo las denominaciones de aloja y añapa se definen bebidas que difieren en la materia prima utilizada y no en el producto final obtenido (Biurrun et al. 2007).

Algunos productos presentan denominaciones similares a las que reciben en Cuyo y Chile, y aunque se trate de alimentos o bebidas diferentes, estas semejanzas en el nombre podrían estar estableciendo orígenes comunes o tal vez propiedades físicas parecidas. Un ejemplo de ello está dado por la "chupilca", que en el área es el nombre de una bebida elaborada mezclando coñac con huevo o a su variante de ñaco con vino y azúcar, mientras que en Mendoza se denomina "chipilca" a un alimento que se logra mezclando ñaco con agua hirviendo y azúcar (Agüero Blanch 1967). Por otra parte, en Chile se denomina "cupilca" o "chupilca" a una mezcla de harina remojada en vino (Alarcón y Nahuelcheo 2008). Además son para destacar las similitudes existentes entre los ingredientes que intervienen en la "chipilca" de Mendoza, la "chupilca" de Chile y la "curillena" mencionada en este trabajo. Todas estas similitudes entre denominaciones e ingredientes permiten suponer que se trata de bebidas o alimentos con un origen común que responden a variantes locales de acuerdo a cada región. Esto demuestra un proceso de continuidad cultural en el oeste de La Pampa que se fundamenta en el origen poblacional de la zona.

La información registrada demuestra una falta de correlación entre el conocimiento de las plantas y su uso. Concretamente existe un conocimiento mayor de las plantas alimenticias que las que realmente se utilizan y esto se advierte mejor entre los pobladores de edad más avanzada. Este proceso de pérdida se relaciona con cambios en la forma de vida, que se manifiestan a través de la substitución de alimentos recolectados por productos manufacturados del mercado (Arenas y Scarpa 2007; Hilgert 1999; Ladio y Lozada 2001; Reyes-García et al. 2005). Buenos ejemplos de lo mencionado son los reemplazos de algunas variantes de arropes por mermeladas y de la aloja y la chupilca por bebidas alcohólicas comerciales. Esta situación pone en evidencia lo que ya se ha señalado en otros trabajos a escala mundial. En tal sentido, el panorama actual de la alimentación en la sociedad moderna globalizada muestra una homogeneización cultural que en ocasiones pone en riesgo la subsistencia de la alimentación tradicional junto a otros elementos que hacen a la identidad de los pueblos (Castro Díaz 2005; Kuhnlein y Receveur 1996). Estos hechos destacan la importancia de los estudios etnobiológicos en pos del rescate de esos conocimientos.

\section{Conclusión}

Se logró un registro exhaustivo de los recursos vegetales utilizados en el área como fuente de alimento que alcanzó un total de 62 taxones vegetales, dentro de los cuales el $23 \%$ está constituido por plantas silvestres. En relación con su origen, el $30 \%$ está representado por especies nativas y en este grupo se detectaron nueve endemismos. Se documentaron también sus nombres vernáculos y los principales platos y bebidas que establecen puntos en común y diferencias con otras sociedades.

La dieta de la población es fundamentalmente carnívora. La mayor parte de los platos principales tienen como ingrediente principal la carne de animales domésticos o silvestres y en cuya elaboración las plantas cumplen un rol complementario. La recolección de productos vegetales para la alimentación por parte de los pobladores es escasa. La participación de los vegetales en la dieta está representada principalmente por especies adquiridas comercialmente, algunas de las cuales también suelen ser cultivadas en los huertos hogareños.

Se evidencia la disminución del uso de algunos recursos vegetales silvestres en la alimentación así como la pérdida de la elaboración de conservas de alimentos con productos vegetales recolectados que estaban bien representados en el pasado. En ambos casos el proceso se ve facilitado por la substitución con productos alimenticios manufacturados. Esto último, sin embargo, significó una garantía en la provisión de ciertos alimentos, una ampliación de la oferta de productos alimenticios, y la posibilidad de acceder a una dieta más balanceada.

El cultivo de plantas con fines alimentarios también está poco representado en el área, siendo la escasez de agua uno de los factores que más condicionan esta actividad y que se acentúa con la facilidad de adquisición de estos productos en los comercios.

En las estrategias de alimentación de la comunidad aquí estudiada aún persisten tradiciones locales de 
la región pampeana y cuyana que presentan nexos indiscutibles con la cocina aborigen y criolla, esta última a su vez fuertemente influenciada por la cultura gastronómica española. No obstante, se observa una paulatina substitución de platos, insumos y prácticas vinculadas a la alimentación como efecto del ingreso de nuevas pautas culturales hegemónicas.
Agradecimientos: El autor desea agradecer al Licenciado Pastor Arenas Rodríguez por la lectura crítica del manuscrito y a los evaluadores de la revista. Este trabajo fue financiado por la Facultad de Ciencias Exactas y Naturales de la Universidad Nacional de La Pampa.

\section{Referencias Citadas}

Agüero Blanch, V.O. 1967. Supervivencia aborigen en la alimentación, en el departamento Malargüe (Mendoza). Anales de Arqueología y Etnología 22:93-100.

Alarcón, A.M. e Y. Nahuelcheo S. 2008. Creencias sobre el embarazo, parto y puerperio en la mujer mapuche: conversaciones privadas. Chungara Revista de Antropología Chilena 40:193-202.

Arenas, P. y G.F. Scarpa 2007. Edible wild plants of the Chorote indians, Gran Chaco, Argentina. Botanical Journal of the Linnean Society 153:73-85.

Arias Toledo, B., L. Galetto y S. Colantonio 2007. Uso de plantas medicinales y alimenticias según características socioculturales en Villa Los Aromos (Córdoba, Argentina). Kurtziana 33:79-88.

Biurrun, E., L. Galetto, A.M. Anton y F. Biurrun 2007. Plantas silvestres comestibles utilizadas en poblaciones rurales de la provincia de La Rioja (Argentina). Kurtziana 33:121-140.

Böhm de Saurina, K. 1961. Punta del Agua (provincia de Mendoza). Estudio antropológico-social preliminar. Anales de Arqueología y Etnología 16:181-199.

Borrero, L. A. 2002. Arqueología y biogeografía humana en el sur de Mendoza (Comentario crítico). En Entre Montañas y Desiertos. Arqueología del Sur de Mendoza, editado por A. Gil y G. Neme, pp. 195-202. Sociedad Argentina de Antropología, Buenos Aires.

Cabrera, A.L. 1971. Fitogeografía de la República Argentina. Boletín de la Sociedad Argentina de Botánica 14:1-42.

Capparelli, A. 2007. Los productos alimenticios derivados de Prosopis chilensis (Mol.) Stuntz y P. flexuosa DC. Fabaceae, en la vida cotidiana de los habitantes del NOA y su paralelismo con el algarrobo europeo. Kurtziana 33:103-119.

Castro Díaz, E. 2005. Food sovereignty and traditional knowledge. International Workshop on Traditional Knowledge. United Nations, Panamá.

Comerci, M.E. 2008. Construcción social del extremo oeste pampeano: tiempos, espacios y sujetos. En Trabajo, Propiedad y Tecnología en la Argentina rural del siglo XX. V Jornadas de Investigación y Debate, pp. 1-22. Universidad Nacional de Quilmes, Quilmes.

Covas, M.R. 1998. Los espacios socioeconómicos de la provincia de La Pampa (Argentina). Huellas 3:11-27.

Hilgert, N.I. 1999. Las plantas comestibles en un sector de las Yungas meridionales (Argentina). Anales del Jardín Botánico de Madrid 57:117-138.
INTA, Universidad Nacional de La Pampa, Gobierno de La Pampa 2004. Inventario Integrado de los Recursos Naturales de la Provincia de La Pampa. Clima, Geomorfología, suelo Vegetación y fauna de Vertebrados. Segunda edición, INTA, UNLPam, Gob. La Pampa. Santa Rosa.

Jolís, J. 1972 [1789]. Ensayo sobre la Historia Natural del Gran Chaco. Facultad de Humanidades. Universidad Nacional del Nordeste, Chaco.

Kuhnlein, H.V. y O. Receveur 1996. Dietary change and traditional food systems of indigenous people. Annual Review of Nutrition 16:417-442.

Ladio, A. 2002. Las Plantas Silvestres Comestibles en el Noroeste Patagónico y su Utilización por las Poblaciones Humanas: una Aproximación Cuantitativa. Tesis Doctoral. Universidad Nacional del Comahue, Bariloche.

- - - 2004. El uso actual de plantas nativas silvestres y comestibles en poblaciones mapuches del NO de la Patagonia. Boletín Latinoamericano y del Caribe de Plantas Medicinales y Aromáticas 3:30-34.

Ladio, A.H. y M. Lozada 2001. Nontimber forest product use in two human populations from Northwest Patagonia: A quantitative approach. Human Ecology 29:367-380.

Martin, G.J. 2000. Etnobotánica. Manual de Métodos. Manuales de conservación. Serie Pueblos y Plantas 1. World Wildlife Foundation. Organización de las Naciones Unidas para la Educación, la Ciencia y la Cultura y Royal Botanic Garden, Kew. Nordan - Comunidad, Montevideo.

Martinelli, M. (coord.) 2008. Sabores de Nuestros Pueblos: Usos de los Recursos Naturales en la Alimentación y Comidas Tradicionales en las Comunidades de Valle Fértil, San Juan. Universidad Nacional de San Juan, San Juan.

Martínez Carretero, E. 2004. La provincia fitogeográfica de la Payunia. Boletín de la Sociedad Argentina de Botánica 39:195-226.

Martínez Crovetto, R. 1968. Estudios etnobotánicos III. Nombres de plantas y su utilidad, según los indios araucano-pampas del oeste de Buenos Aires (República Argentina). Etnobiológica 12:1-24.

- - - 1982. Breve panorama de las plantas utilizadas por los indios de Patagonia y Tierra del Fuego. Suplemento Antropológico 17:61-97.

Martínez Sarasola, C. 1992. Nuestros Paisanos los Indios. Vida, Historia y Destino de las Comunidades Indígenas en la Argentina. EMECÉ, Buenos Aires. 
Muiño, W.A. 2010a. El uso de las Plantas Silvestres por la Comunidad de Chos Malal (Provincia de La Pampa). Tesis para acceder al grado de Doctor en Ciencias Naturales. Facultad de Ciencias Naturales y Museo, Universidad Nacional de La Plata, La Plata.

- - - 2010b. Ethnobotanical study of the rural population of the west of the Pampa plain (Argentina). Ethnobotany Research \& Applications 8:219-231.

Prieto, M.R. 1997-98. Formación y consolidación de una sociedad en un área marginal del Reino de Chile. En La provincia de Cuyo en el siglo XVII. Tesis Doctoral. Anales de Arqueología y Etnología 52/53:313-327.

Rapoport, E.H., A.H. Ladio y E.H. Sanz 2001. Plantas Nativas Comestibles de la Patagonia Andina. I. Ediciones Imaginaria, Bariloche.

Remedi, F.J. 1997. Las condiciones de vida material: cocinas étnicas y consumo alimentario en la provincia de Córdoba a comienzos de siglo. En Procesos Socioculturales y Alimentación, compilado por M. Álvarez y L. Pinotti, pp. 99-137. Ed. del Sol, Buenos Aires.

Reyes-García, V., V. Vadez, T. Huanca, W. Leonard y D. Wilkie 2005. Knowledge and consumption of wild plants: a comparative study in two Tsimane' villages in the Bolivian Amazon. Ethnobotany Research \& Applications 3:201-207.

Ruiz Leal, A. 1972. Flora popular mendocina. Deserta 3:3-296.

Scarpa, G. F. 1999. El arrope en el noroeste argentino. Ayer una fiesta, hoy un capital. En Los Sabores de España y América.
Cultura y Alimentación, compilado por A. Garrido Aranda, pp. 95-139. La Val de Onsera, Huesca.

Scarpa, G.F. 2009. Wild food plants used by the indigenous peoples of the South American Gran Chaco: A general synopsis and intercultural comparison. Journal of Applied Botany and food Quality 83:90-101.

Scarpa, G.F. y P. Arenas 1996. Especias y colorantes en la cocina tradicional de la Puna jujeña (Argentina). Candollea 51:483-514.

Steibel, P.E. 1997. Nombres y usos de las plantas aplicados por los indios ranqueles de La Pampa (Argentina). Revista de la Facultad de Agronomía Universidad Nacional de La Pampa. 9:1-40.

Troiani, H., P. Steibel, A. Prina y G. Alfonso 1994. Catálogo preliminar de la flora de la provincia de La Pampa. Manuscrito en posesión de los autores.

Zuloaga, F.O., O. Morrone y M.J. Belgrano (eds.) 2008. Catálogo de las Plantas Vasculares del Cono Sur (Argentina, sur de Brasil, Chile, Paraguay y Uruguay). Missouri Botanical Garden Press. Missouri.

\section{Sitios WEB}

Instituto Nacional de Estadísticas y Censos (INDEC). 2001. Censo nacional de población, hogares y vivienda. Serie 2. Resultados generales. $\mathrm{N}^{\circ} 11$ Provincia de La Pampa. http://www.indec. gov.ar/websenso/provincias_2/provincias.asp (5 mayo 2011).

United States Department of Agriculture. Natural Resources Conservation Service. (2011). Plants database. http://plants. usda.gov/java/index.jsp (2 marzo 2011). 